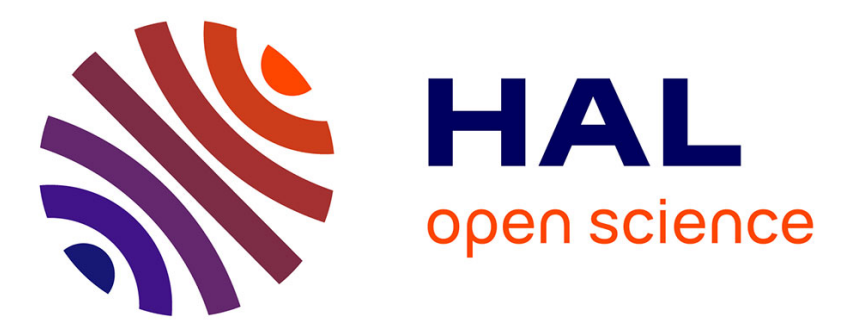

\title{
Increasing life science resources re-usability using Semantic Web technologies
}

Marine Louarn, Fabrice Chatonnet, Xavier Garnier, Thierry Fest, Anne

Siegel, Olivier Dameron

\section{- To cite this version:}

Marine Louarn, Fabrice Chatonnet, Xavier Garnier, Thierry Fest, Anne Siegel, et al.. Increasing life science resources re-usability using Semantic Web technologies. eScience 2019 - 15th International eScience Conference, Sep 2019, San Diego, United States. pp.1-9. hal-02274982

\section{HAL Id: hal-02274982 \\ https://hal.inria.fr/hal-02274982}

Submitted on 30 Aug 2019

HAL is a multi-disciplinary open access archive for the deposit and dissemination of scientific research documents, whether they are published or not. The documents may come from teaching and research institutions in France or abroad, or from public or private research centers.
L'archive ouverte pluridisciplinaire HAL, est destinée au dépôt et à la diffusion de documents scientifiques de niveau recherche, publiés ou non, émanant des établissements d'enseignement et de recherche français ou étrangers, des laboratoires publics ou privés. 


\section{Increasing life science resources re-usability using Semantic Web technologies}

\author{
Marine Louarn* \\ MICMAC - UMR_SI236\& IRISA \\ INSERM \& Univ Rennes, Inria, CNRS, \\ Rennes, France \\ marine.louarn@irisa.fr \\ Thierry Fest \\ MICMAC - UMR_S1236 \\ INSERM, Univ Rennes, CHU Rennes, EFS \\ Rennes, France \\ thierry.fest@univ-rennes1.fr
}

\author{
Fabrice Chatonnet* \\ MICMAC - UMR_S1236 \\ Rennes, France \\ fabrice.chatonnet@chu-rennes.fr \\ Anne Siegel \\ IRISA \\ Univ Rennes, Inria, CNRS, IRISA \\ Rennes, France \\ anne.siegel@irisa.fr
}

IRISA INSERM, Univ Rennes, CHU Rennes, EFS Univ Rennes, Inria, CNRS, IRISA

\author{
Olivier Dameron \\ IRISA \\ Univ Rennes, Inria, CNRS, IRISA \\ Rennes, France \\ olivier.dameron@univ-rennes1.fr
}

* These authors contributed equally to this paper

\begin{abstract}
In life sciences, current standardization and integration efforts are directed towards reference data and knowledge bases. However, original studies results are generally provided in non standardized and specific formats. In addition, the only formalization of analysis pipelines is often limited to textual descriptions in the method sections. Both factors impair the results reproducibility, their maintenance and their reuse for advancing other studies. Semantic Web technologies have proven their efficiency for facilitating the integration and reuse of reference data and knowledge bases.

We thus hypothesize that Semantic Web technologies also facilitate reproducibility and reuse of life sciences studies involving pipelines that compute associations between entities according to intermediary relations and dependencies.

In order to assess this hypothesis, we considered a case-study in systems biology (http://regulatorycircuits.org), which provides tissue-specific regulatory interaction networks to elucidate perturbations across complex diseases. Our approach consisted in surveying the complete set of provided supplementary files to reveal the underlying structure between the biological entities described in the data. We relied on this structure and used Semantic Web technologies (i) to integrate the Regulatory Circuits data, and (ii) to formalize the analysis pipeline as SPARQL queries.

Our result was a $335,429,988$ triples dataset on which two SPARQL queries were sufficient to extract each single tissuespecific regulatory network.
\end{abstract}

Index Terms-Semantic Web, bioinformatics, SPARQL, reproducibility of results

\section{INTRODUCTION}

There are more than 1500 life science databases, each able to answer important questions in a particular domain [1]. Most of them offer a dedicated repository for expert knowledge but they fail at structuring biological datasets [2]. Indeed, the classical data management technologies used by the life science community range from data storage in the form of multiple tabulated files analyzed with spreadsheets, silo models in complex database management systems with a predetermined scheme of federated data such as Intermine [3] or Biomart [4], to ad-hoc community centralized models such as in bio-imaging communities. These solutions address immediate integration requirements but they are poorly compatible with scalable and flexible integration needs, either between communities (for example to jointly analyze medical imaging and genomics data) or with the world of linked data to enrich analyses with symbolic knowledge selected in a fine and contextual way in existing databases.

An alternative approach for structuring and analyzing heterogeneous datasets and knowledge bases is based on the Semantic Web technologies. They are an extension of the current Web that provides an infrastructure for integrating data and metadata in order to support unified reasoning and querying as a virtual unified dataset [5]. This approach has been widely adopted by the life science community for releasing reference data and knowledge bases [6], [7] in RDF triplestores. Thanks to the growth of linked data, supported by the Linked Open Data initiative (LOD) [8], more and more reference data and knowledge bases are integrated. Moreover, it also evolved into the FAIR principles for ensuring that the available data are Findable, Accessible, Interoperable and Reusable [9]-[12]. A bottleneck for a broader adoption of these technologies by the life science community is a technical barrier: a complete analysis scheme based on Semantic Web technologies requires users first to prepare their data according to a RDF framework to make them exploitable, and second to become familiar with the SPARQL language either for querying their own RDF data, or for analyzing them in relation with the other triplestores of the LOD. A second barrier is given by the concept of "pipeline" and workflow which is widely used in life sciences: data analysis of large-scale data consists of score computation chains involving several files to produce final results. A classical example of such an analysis pipeline is given by the Regulatory Circuits [13] project (http://regulatorycircuits.org), which consists of several analyses on heterogeneous and multi- 
layer "omics" data on human patient cells from many tissues. The output of the Regulatory Circuits study is a family of scored tissue-specific regulatory interaction networks that can be explored through text files. The method used to obtain this output is described in supplementary materials. The associated computational scripts and algorithms are limited to the considered dataset. Moreover, feedback from the Regulatory Circuits' authors was limited when solicited about the methodology. This has a huge impact on i) the reproducibility of the results, ii) their maintenance as they will need to be updated when newer or additional data sources are released and iii) their reuse for advancing other studies (which was the reason these results were generated in the first place). In addition, the output format makes it impossible to explore and enrich the data by combining them to additional knowledge on entities stored in LOD public databases.

In this article, we introduce an approach based on Semantic Web technologies to revisit the analysis workflow performed in the Regulatory Circuits study. We propose an RDF representation of the unstructured data files in order to exhibit links (triples) between biological products associated with standardized identifiers in the dataset. Based on this RDF representation of the dataset, we show that the output of the Regulatory Circuits study can be obtained by two SPARQL queries. The article is organized as follows. Section II details the application field of cellular regulation in life sciences and the main international projects producing reference datasets in this field. Section III describes our approach for structuring and integrating the Regulatory Circuits datasets: relevant files identification, data structuring, data integration, query construction. Section IV is a discussion and a conclusion about our approach benefits and limitations.

\section{BACKGROUND}

In biological sciences, gene regulation (also called transcriptional regulation) is a major field of investigation. It allows a better understanding of major processes such as cell differentiation (how to obtain one or several effective cell types from a common progenitor cell), cell identity (how gene expression is used to define a specific cell type) and cell transformation (how altered gene expression can lead to cell death or cancer). To understand gene expression regulation in a specific context, one needs to perform diverse types of whole genome spanning experiments, currently made available by the recent advent of high throughput sequencing. However, these experiments usually produce huge amounts of data which are heterogeneous by nature, not well organized, not well structured, and not linked to other data or to reference knowledge.

To construct gene regulation networks, one has to associate target genes with their potential regulators, so-called transcription factors (TF). At the DNA level, a TF will bind to a definite sequence (called a binding motif) in a specific regulatory region, which should be in an opened $3 \mathrm{D}$ conformation to allow the regulation (Fig. 1), and which can be located close or far from its target gene. This binding event will then initiate a cascade of molecular events eventually leading to regulation (induction or inhibition) of the target gene expression. Researchers in life sciences and in bioinformatics use huge amounts of data to build extensive regulatory networks from these different entities (genes, TF, regulatory regions), mainly by statistical and machine learning methods. This has been the case with data integration performed on the recent releases of massive databases from the ENCODE [14] [15], FANTOM5 [16] [17] and RoadMap Epigenomics [18] [19] consortia. These data are usually released as primary raw datasets, usable processed data or compiled networks but with few possibilities for adding easily new links between the data or for re-using the published bioinformatics pipelines. Consequently, those datasets have no or low compliance to the FAIR guidelines [10]. ENCODE data for example have only been published as ontologies [20], processed data together with scripts used to obtain them, or unlinked datasets. Although part of FANTOM5 has been released under the RDF format, it only concerns gene expressions and not regulatory data [16], [21].

One of the most recent example was the deep exploitation of the FANTOM5 datasets to establish 394 cell-type specific regulatory networks [13]. Contrasting with previous attempts, these networks were not derived from a statistical analysis of biological measurements but based on a set of computed correlations between regulatory regions, gene expression, and curated and scored TF binding sites. Datasets were published either as input (raw data) or intermediary (authors-processed) data files, in the form of tabulation or comma-delimited data files with various format and contents (see Table I). That dataset of regulatory networks is amongst the most recent and the most complete. They are therefore of great potential interest for the community, so we decided to transform the study original data and results to make them more easily available and usable. To this end, we identified the useful data, we structured them according to a schema supporting the network building task and integrated them in order to be able to query them. Query results could then be interpreted as the Regulatory Circuits cell-type specific networks.

\section{CONTRIBUTION}

Semantic Web technologies provide the infrastructure for integrating, combining with knowledge bases and querying data. They have been successfully applied on reference data, that are arguably the most prone to be reused. We have seen that this requirement also applies to research results, such as the ones from the Regulatory Circuits study. There are some ongoing efforts in the neuroimaging community to use Semantic Web technology for sharing and reusing datasets [22], but these are not directly applicable to our situation.

By structuring and integrating the data from Regulatory Circuits we were aiming at efficiently recovering the TF-gene relationships computed in the original work. We also wanted to make the data structure easily extendable to new data for the users. To do so, a requirement was to identify all the necessary entities from the published datasets (files) and the relevant 
steps of the pipeline necessary for deriving the relations between genes and transcription factors. We reused the rank values for the expression measures because the Regulatory Circuits' method section does not specify how to compute them, as well as the distance values between enhancers and transcripts. We computed the other elements.

\section{A. Identifying relevant files among all Regulatory Circuits resources}

The first step of data structuring was the identification of all the necessary files from Regulatory Circuits (available from the supplementary archive file at http://regulatorycircuits.org), including raw data (input) and pre-processed (intermediary) files, to recreate the published pipeline. Table I presents a review of the supplementary files in Regulatory Circuits, including the number of headers and comment lines, the entity names, and their format. Regulatory Circuits files also contained the computed networks, available on the download tab of their website under the Networks category. FANTOM5_individual_networks.tar contained 394 tissues-specific networks and Network_compendium.zip contained 32 highlevel networks and 40 public ones. We did not use those to construct our model.

On the 21 files present in Table I, fourteen were input files and seven were intermediary ones. The dataset was composed of text files of various size ranging from 184 to $124,358,159$ lines and from 3 columns to 890 . This lead to large files which were complex to explore and made retrieving specific information difficult. For example the file ho19.cage_peak_OK.txt was just over 1.1GB.

These files had heterogeneous structures of headers and entities identifiers. 5 files had no header and one had 3 header lines. 3 of the files with headers were mis-formatted (enhancer_expr.rank.txt, promoter_expr.rank.prec90.txt and transcript_expr.rank.prec90.txt). They had an offset of 1 between the number of columns in the header versus in the data, which forced us to retrieve the data of the $(n+1)^{\text {th }}$ column to get the information related to the $\mathrm{n}^{\text {th }}$ element. This contributeed to the complexity of navigating those files. One file also had 800 comment lines above file header and one (motif_defs.txt) which contained only comments and non-formatted text.

To increase the difficulty of links retrieval between the files, the entity identifiers were not homogeneous across the dataset. For example, the promoter regions had an identifier sometimes following the pattern: chr:start-end,strand and some other times following: p@chr:start..end,strand (with chr being the chromosome on which is the region and start and end are its chromosomal locations). Samples names also differed across files headers. The most common denomination was the libld identifier based on $C N h s+n b$ where $n b$ is a five digit integer (e.g.: CNhs11051), but in hg19_permissive_enhancers_expression_rle_tpm.csv the sample name were cellType + donor $+n b$ : libld with cellType either only the cell line or the cell line and the localization (example: Adipocyte - breast donorl : CNhs11051). In hg19.cage_peak_coord_robust.bed this identifier were tpm.Adipocyte\%20\%20breast\%2c\%20donor $1 . C N h s 11051.11376-118$ A8.

To identify which files were necessary to rebuild the regulation networks, we mapped the entities and files on the biological background (see section II) as shown in Figure 1.

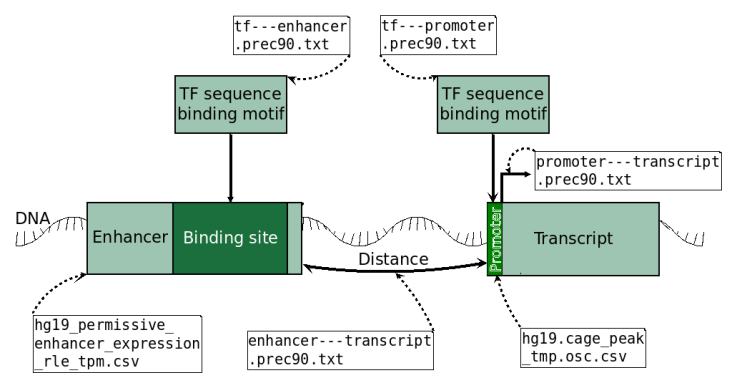

Fig. 1: Underlying biological background used to infer TFgene relationships by the Regulatory circuits pipeline. TF can interact with enhancers or promoters binding sites. Regulatory Circuits original files containing the necessary entities and relations are also represented.

For the attributes linked to entities, only elements that improved the recovery of entities by users were kept, such as: binding sites motif for transcription factors, ENSEMBL identifiers for genes and transcripts and DNA strands on which promoters are located.

\section{B. Structuration}

Once we identified all the files, entities and relations required to build TF-genes interactions, we structured their content. To do so, we first placed the entities in a graph as shown in Figure 2a. The only attributes given at this step were the expression levels of enhancers and promoters.

Second, we retrieved the relationships given in the Regulatory Circuits data. For those, we used the pre-computed distances between the transcripts and the regulatory regions (enhancers and promoters), as well as the weight of the transcripts-enhancer distances. We also kept all the preprocessed confidence scores for the transcription factors / regions interactions. In the Regulatory Circuits article, the authors used a rank normalization of their expressions data in the final pipeline, so we used their intermediary files including these ranks for the enhancers and promoters expressions. We also kept the file including the rank for the transcript. All these interactions are described in Figure $2 b$.

Third, the structure built from all these data and their interactions allowed us to easily retrieve the TF-gene relationships by navigating through the entities and their relations (Figure 2c).

\section{Integration}

Once the data had been structured, we integrated them so that they can be browsed and queried. To do so, we unified the identifiers and explicited the links between the entities.

We created a new set of rules to homogenize the entities identifiers across files ni order to facilitate integration. 
TABLE I: Regulatory Circuits files' review

\begin{tabular}{|c|c|c|c|c|c|c|c|c|c|c|c|c|}
\hline type of file & file name & format & $\begin{array}{c}\text { header } \\
\text { lines }\end{array}$ & $\begin{array}{c}\text { missformated } \\
\text { header }\end{array}$ & $\begin{array}{l}\text { comment } \\
\text { lines }\end{array}$ & $\begin{array}{l}\text { data } \\
\text { lines }\end{array}$ & $\begin{array}{c}\text { nb } \\
\text { columns }\end{array}$ & $\begin{array}{l}\text { Label of column(s) } \\
\text { with ID }\end{array}$ & ID format & Entities & Source & content \\
\hline Input & $\begin{array}{c}\text { hg19 } \\
\text { permissive } \\
\text { enhancers } \\
\text { expression } \\
\text { rle } \\
\text { tpm.csv }\end{array}$ & $\operatorname{csv}()$, & 1 & & 0 & 43011 & 809 & 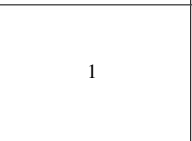 & chr:start-end & enhancer & [1] & [a] \\
\hline data & $\begin{array}{l}\text { permissive } \\
\text { enhancers.bed }\end{array}$ & bed12 (tab-delim) & 1 & & 0 & 43011 & 12 & 4 & chr:start-end & enhancer & [1] & [b] \\
\hline for & $\begin{array}{c}\text { robust } \\
\text { enhancers.bed }\end{array}$ & bed12 (tab-delim) & 1 & & 0 & 38554 & 12 & 4 & chr:start-end & enhancer & [1] & {$[\mathrm{b}]$} \\
\hline network & $\begin{array}{c}\text { hg19.cage } \\
\text { peak } \\
\text { tpm.osc.txt }\end{array}$ & tab-delim & 3 & & 893 & 184827 & 890 & 1 & chr:start-end,strand & promoter & [2] & [a] \\
\hline \multirow[t]{13}{*}{ inference } & $\begin{array}{c}\text { hil9.cage } \\
\text { peak } \\
\text { coord } \\
\text { robust.bed }\end{array}$ & bed12 (tab-delim) & 0 & & 0 & 184827 & 12 & 4 & chr:start-end,strand & promoter & [2] & [b] \\
\hline & $\begin{array}{c}\text { gene } \\
\text { coord.bed }\end{array}$ & bed6 (tab-delim) & 0 & & 0 & 19125 & 6 & 4 & GENE_SYMBOL & gene & [3] & {$[\mathrm{b}]$} \\
\hline & gene_ids.txt & tab-delim & 1 & & 0 & 19125 & 3 & $\frac{1}{2}$ & $\begin{array}{l}\text { ENSG00000000000 } \\
\text { GENE SYMBOL }\end{array}$ & $\begin{array}{l}\text { gene } \\
\text { gene }\end{array}$ & [3] & [c] \\
\hline & & & & & & & & $\frac{2}{3}$ & $\frac{\text { GENE_SYMBOL }}{\text { EntrezID }}$ & $\begin{array}{l}\text { gene } \\
\text { gene }\end{array}$ & & \\
\hline & $\begin{array}{c}\text { mhc } \\
\text { genes.txt }\end{array}$ & tab-delim & 1 & & 0 & 184 & 1 & 1 & GENE_SYMBOL & gene & [3] & [c] \\
\hline & $\begin{array}{l}\text { transcript } \\
\text { cooord.bed }\end{array}$ & bed6 (tab-delim) & 0 & & 0 & 53449 & 6 & 4 & GENE _SYMBOL-000 & transcript & [3] & {$[\mathrm{b}]$} \\
\hline & \multirow{2}{*}{$\begin{array}{l}\text { transcript- } \\
\text { gene.txt }\end{array}$} & \multirow[t]{2}{*}{ tab-delim } & \multirow[t]{2}{*}{1} & & \multirow[t]{2}{*}{0} & \multirow[t]{2}{*}{53449} & \multirow[t]{2}{*}{4} & 1 & GENE_SYMBOL-000 & transcript & \multirow[t]{2}{*}{ [3] } & \multirow[t]{2}{*}{ [c] } \\
\hline & & & & & & & & $\begin{array}{l}2 \\
3 \\
4\end{array}$ & $\begin{array}{c}\text { GENE_SYMBOL } \\
\text { ENSG00000000000 } \\
\text { ENST00000000000 }\end{array}$ & $\begin{array}{l}\text { gene } \\
\text { transcript } \\
\text { gene }\end{array}$ & & \\
\hline & $\begin{array}{c}\text { tss } \\
\text { coord.bed }\end{array}$ & bed6 (tab-delim) & 0 & & 0 & 53449 & 6 & 4 & GENE_SYMBOL-000 & gene & [3] & [b] \\
\hline & $\begin{array}{c}\text { motif } \\
\text { _defs.txt }\end{array}$ & space-delim & 0 & & 1772 & N/A & N/A & N/A & N/A & & [4] & {$[\mathrm{g}]$} \\
\hline & $\begin{array}{c}\text { motif } \\
\text { instances.bed }\end{array}$ & bed6 (tab-delim & 0 & & 0 & 124358159 & 6 & 4 & TF _0 & $\mathrm{TF}$ & [4] & {$[\mathrm{b}]$} \\
\hline & \multirow{2}{*}{ 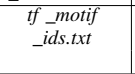 } & \multirow[t]{2}{*}{ tab-delim } & \multirow[t]{2}{*}{1} & & \multirow[t]{2}{*}{0} & \multirow[t]{2}{*}{1792} & \multirow[t]{2}{*}{3} & 1 & $\mathrm{TF}$ & TF & \multirow[t]{2}{*}{ [4] } & \multirow[t]{2}{*}[\mathrm{g}]{} \\
\hline & & & & & & & & 2 & TF_0 & TF & & \\
\hline \multirow{11}{*}{ files } & $\begin{array}{c}\text { enhancer } \\
\text { expr.rank.txt }\end{array}$ & tab-delim & 1 & $\mathrm{x}$ & 0 & 43011 & 809 & 1 & e@chr:start..end & enhancer & {$[5]^{*}$} & [d] \\
\hline & $\begin{array}{l}\text { enhancer- } \\
\text { transcript } \\
\text {.prec } 90 . t x t\end{array}$ & tab-delim & 1 & & 0 & 950513 & 5 & 1 & e@ @chr:start...nd & enhancer & \multirow[t]{2}{*}[5]{$^{*}$} & \multirow[t]{2}{*}{ [e] } \\
\hline & & & & & & & & $\frac{2}{5}$ & $\begin{array}{c}\text { GENE_SYMBOL-000 } \\
\text { GENE_SYMBOL }\end{array}$ & $\frac{\text { transcript }}{\text { gene }}$ & & \\
\hline & $\begin{array}{c}\text { promoter } \\
\text { _exprank } \\
\text {.prec90.txt } \\
\end{array}$ & tab-delim & 1 & $\mathrm{x}$ & 0 & 59126 & 809 & 1 & p@chr:start..end,strand & promorer & {$[5]^{*}$} & [d] \\
\hline & \multirow[t]{2}{*}{$\begin{array}{l}\text { promoter- } \\
\text { transcript } \\
\text {.prec } 90 . t x t\end{array}$} & \multirow[t]{2}{*}{ tab-delim } & \multirow[t]{2}{*}{1} & & \multirow[t]{2}{*}{0} & \multirow[t]{2}{*}{123440} & \multirow[t]{2}{*}{4} & 1 & p@ chr:start..end,strand & promoter & \multirow[t]{2}{*}{ [5]* } & \multirow[t]{2}{*}{ [e] } \\
\hline & & & & & & & & $\frac{2}{4}$ & $\begin{array}{c}\text { GENE_SYMBOL-000 } \\
\text { GENE_SYMBOL }\end{array}$ & $\frac{\text { transcript }}{\text { gene }}$ & & \\
\hline & $\begin{array}{l}\text { tf-enhancer } \\
\text { precop txt }\end{array}$ & tab-delim & 1 & & 0 & 524816 & 3 & 1 & $\mathrm{TF}$ & $\mathrm{TF}$ & {$[5]^{*}$} & [f] \\
\hline & & & & & & & & 2 & e@ @chr:start..end & enhancer & & \\
\hline & if-promoter & tab-delim & 1 & & 0 & 1169797 & 3 & 1 & $\mathrm{TF}$ & $\mathrm{TF}$ & {$[5]^{*}$} & [f] \\
\hline & & & & & & & & 2 & $\mathrm{p} @$ @ chr:start..end,strand & promoter & & \\
\hline & $\begin{array}{l}\text { transcript } \\
\text {-exprrank } \\
. \text { prec90.txt }\end{array}$ & tab-delim & 1 & $\mathrm{x}$ & 0 & 43352 & 809 & 1 & GENE_SYMBOL-000 & trancript & {$[5]^{*}$} & [d] \\
\hline
\end{tabular}

[1]http://enhancer.binf.ku.dk/Pre-defined tracks.html, [2] http://fantom.gsc.riken.jp/5/datafiles/latest/extra/CAGE peaks/, [3] EnsembI biomart, [4]Pouya Kheradpour (pouyak <a> mit.edu), [5]* Regulatory Circuits: auto produced, [a] Normalized activities,[b] Genomic coordinates, [c] Identifier, [d] Rank of normalized activities,[e] Distances, [f] Confidence score, [g] TF motifs

Regions identifiers were created using the following pattern: $r_{-}$chr $X \_s t a r t \_e n d, r$ being the first letter of the region type ( $e$ for enhancer or $p$ for promoter), $X$ the chromosome number for the region and start and end its chromosomal coordinates. For the expression, we chose to keep the libld identifier (CNhs+nb with $n b$ a five digit integer) of the tissues samples as name and added Rank_ before this identifier for the ranks score of the same samples. Genes, transcripts and $\mathrm{TF}$ retained their original identifiers.

When a relation involved more than two entities or had some attributes, we used reification and represented the relation as an additional entity. The identifier for the reified relation was defined as name1_name $2 \_n b$ with name1 the type of the first entity in the link, name2 the second type and $n b$ a unique integer. The reified relation was then associated to the entities and attributes using regular binary relations (for example in Figure $2 b$, notice that the relation from a TF to a Promoter had a confidence score (confidence); this ternary relation was represented by the tf_promoter entity in the RDF model in Figure 3 which associated a TF, a Promoter and a confidence).

We created a RDF graph of the dataset using Regulatory Circuits data and new entities for representing reified relationships, as shown in Figure 3. The description of entities classes, numbers and attributes in each node can be found in Table II. We integrated these data using RDF and deployed them as 


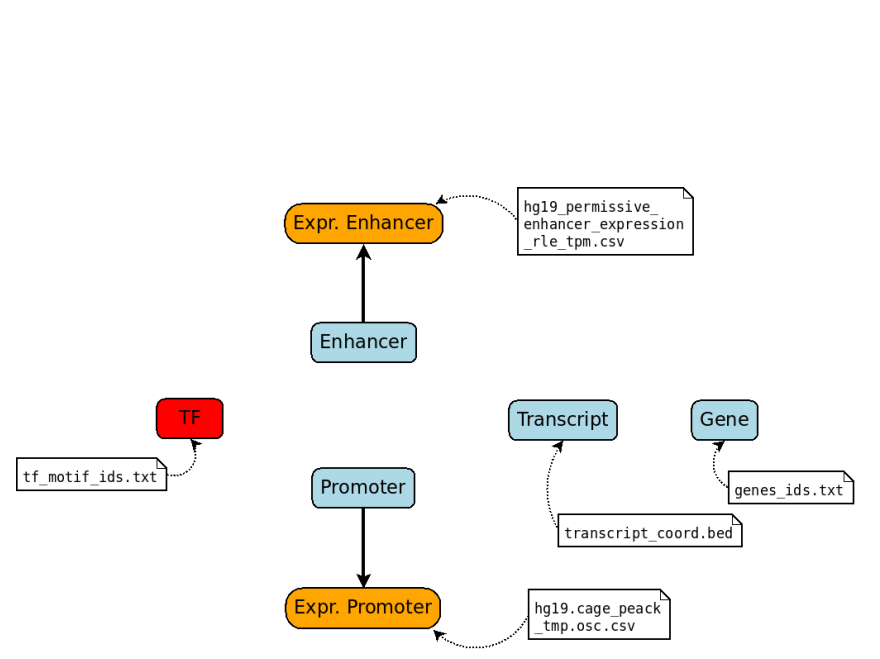

(a) Graph with input files

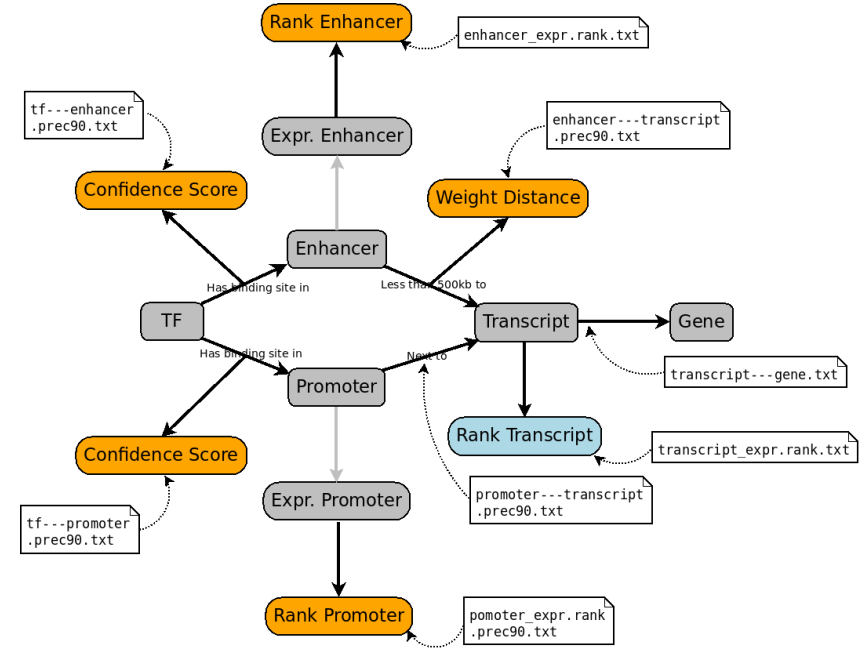

(b) Graph updated with intermediary files

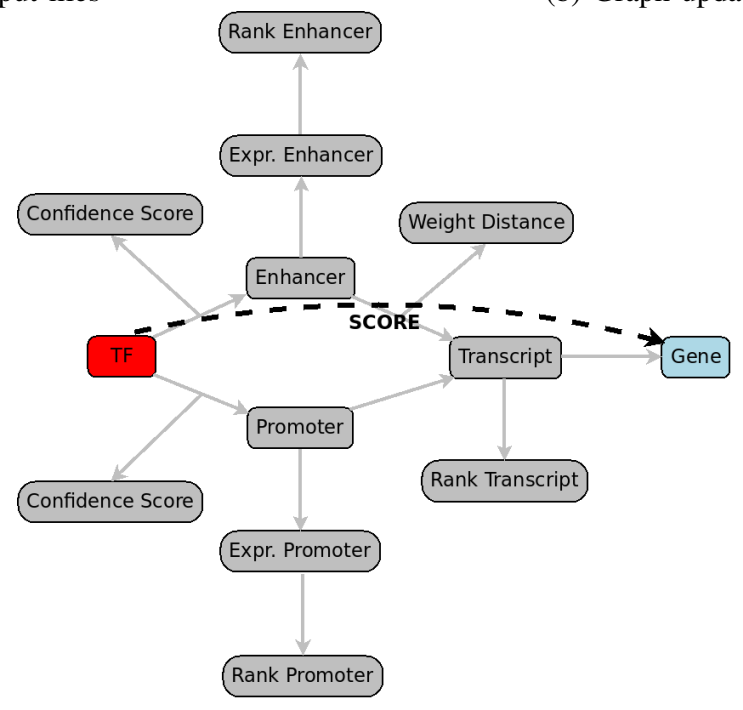

(c) Final structure

Fig. 2: The three steps of data structuring, with identification of the files containing the needed information. In (a) identification of input files. In this step we mostly import entities (genes, TF, regulatory regions). The only imported relation is the expression levels of both types of regulatory regions.

In (b) we added all the information from the intermediary files: interactions between the different elements and scores based on those relations. We also added pre-processed scores on the expression levels, called Ranks.

In (c) we can see that the TF-gene relations were obtained by following the links between entities and that these relations could be weighted using the score from step (b).

a SPARQL endpoint using OpenLink Virtuoso engine.* As shown in Table III, we integrated ten classes representing more than three hundred million triples. To do so, we had to separate some of the files in smaller ones resulting in a total of fortyfour integrated files.

The description of the dataset population can be seen in Figure 3b and Tables II and III: over three million entities, separated in ten classes, each with several attributes.

*The RDF dataset can be retrieved from https://regulatorycircuits-rdf. genouest.org/dump/ and the SPARQL endpoint is accessible at https:// regulatorycircuits-rdf.genouest.org/sparql

\section{Queries}

After integrating all the data, we could query the dataset in order to retrieve the TF-gene relationships for each cell type or tissue. According to Regulatory Circuits there are two ways of getting the transcription factor and gene relationship: using either type of regulatory regions (enhancers or promoters). The first step consisted in computing all the potential TF-gene relations.

The first query used the promoter as the binding region for the TF: starting from the TF, and continuing by the promoter, the transcript and then the gene. To confirm the existence of 


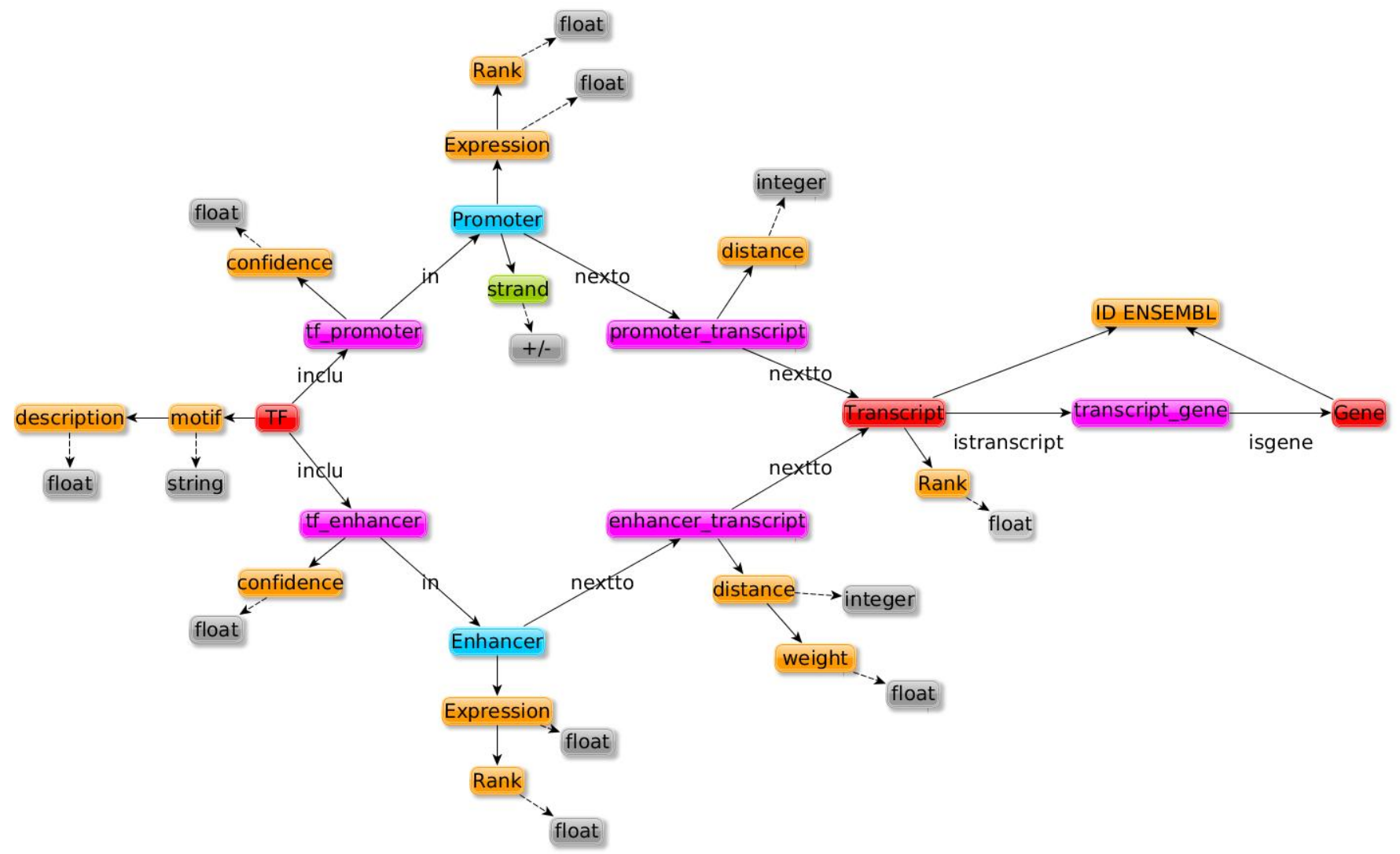

Fig. 3: Data structure after integration. Nodes in red are gene entities, nodes in blue are regulatory region entities and nodes in pink are reified relations. Genomic localization attributes are indicated in green, other attributes are in orange and attributes type are in gray. Details about the number of entities for each nodes can be found in Table II.

this relation, we needed to verify that the TF confidence score and the promoter expression rank were both different from 0.

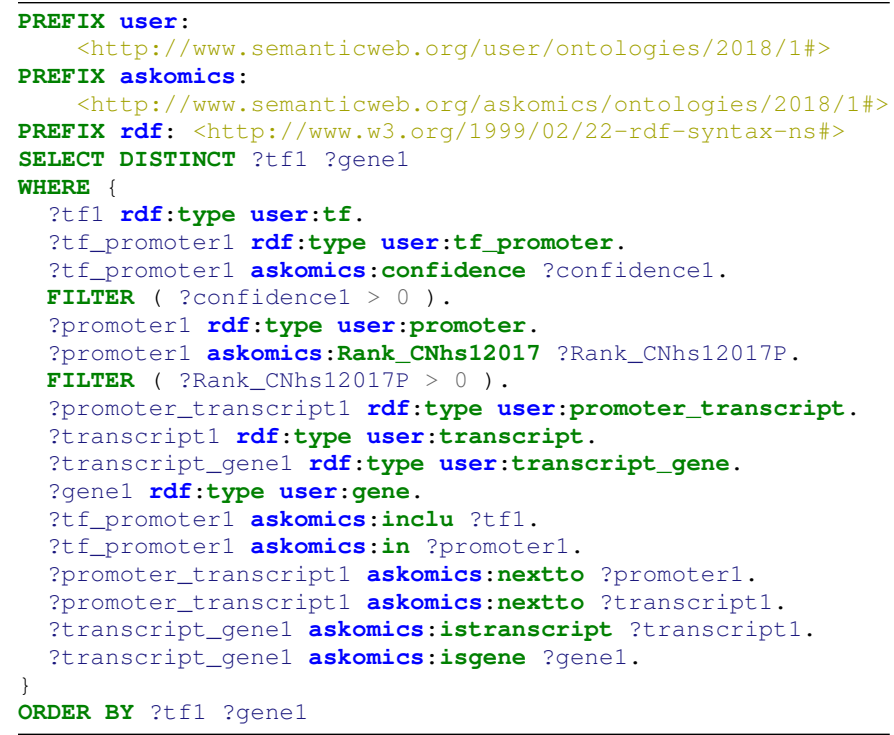

The second query was similar but used the enhancer instead of the promoter: we started from the $\mathrm{TF}$, and proceeded following the enhancer, the transcript and then the gene, making sure that all score component were superior to 0 .

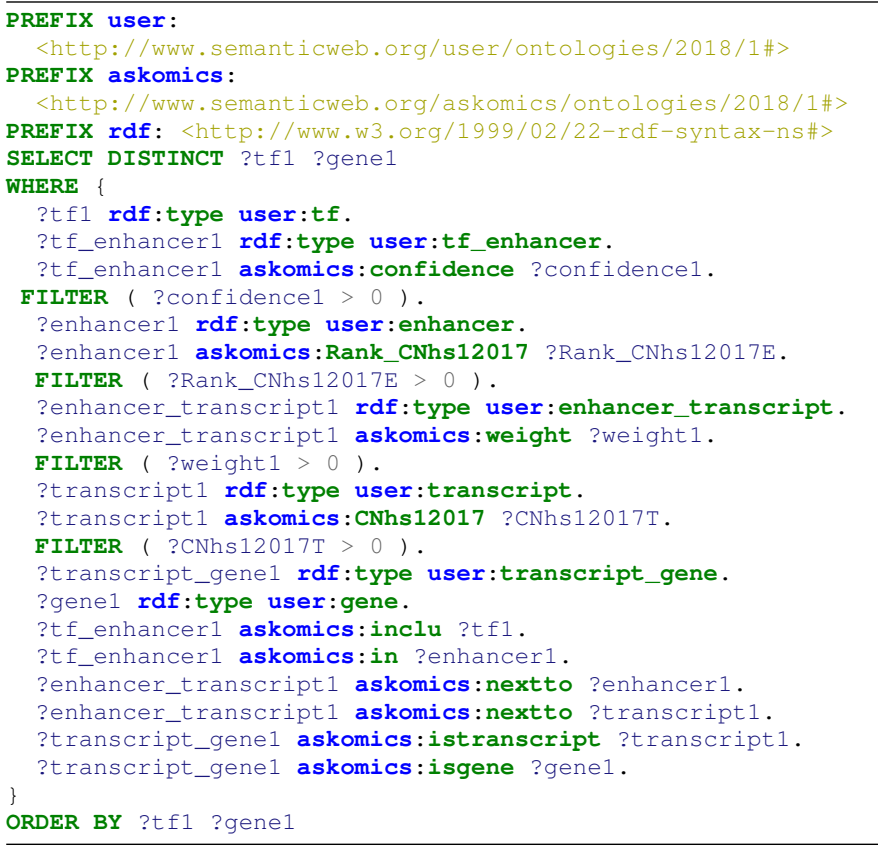


TABLE II: Data population. The first three lines correspond to the nodes in red in Figure 3, the next two correspond to the blue nodes and the last five to the pink ones.

\begin{tabular}{|c|c|}
\hline $\begin{array}{c}\text { Class } \\
\text { (nb of entities) }\end{array}$ & Attributes \\
\hline $\begin{array}{c}\text { Gene } \\
(19125)\end{array}$ & ID_ENSEMBL \\
\hline $\begin{array}{l}\text { Transcript } \\
\text { (53 549) }\end{array}$ & $\begin{array}{c}\text { ID_ENSEMBL } \\
\text { Rank for } 808 \text { pop. }\end{array}$ \\
\hline $\begin{array}{c}\text { TF } \\
(691)\end{array}$ & $\begin{array}{c}\text { motif } \\
\text { description }\end{array}$ \\
\hline $\begin{array}{l}\text { Promoter } \\
(184828)\end{array}$ & $\begin{array}{c}\text { strand } \\
\text { Expression for } 889 \text { pop. } \\
\text { Rank for } 808 \text { pop. }\end{array}$ \\
\hline $\begin{array}{l}\text { Enhancer } \\
\left(\begin{array}{ll}43 & 011)\end{array}\right.\end{array}$ & $\begin{array}{l}\text { Expression for } 808 \text { pop. } \\
\text { Rank for } 808 \text { pop. }\end{array}$ \\
\hline $\begin{array}{l}\text { tf_promoter } \\
\text { (1 1 169 797) }\end{array}$ & $\begin{array}{c}\text { confidence } \\
\text { inclu@tf } \\
\text { in@promoter }\end{array}$ \\
\hline $\begin{array}{l}\text { tf_enhancer } \\
\text { (524 816) }\end{array}$ & $\begin{array}{c}\text { confidence } \\
\text { inclu@tf } \\
\text { in@enhancer }\end{array}$ \\
\hline $\begin{array}{c}\text { transcript_gene } \\
(53449)\end{array}$ & $\begin{array}{c}\text { isgene@gene } \\
\text { istranscript@ transcript }\end{array}$ \\
\hline $\begin{array}{c}\text { promoter_transcript } \\
(123441)\end{array}$ & $\begin{array}{c}\text { distance } \\
\text { nextto@ transcript } \\
\text { nextto@ promoter }\end{array}$ \\
\hline $\begin{array}{c}\text { enhancer_transcript } \\
(950 \text { 514) }\end{array}$ & $\begin{array}{c}\text { distance } \\
\text { weight } \\
\text { nextto@transcript } \\
\text { nextto@promoter }\end{array}$ \\
\hline
\end{tabular}

TABLE III: Integrated data

\begin{tabular}{|c|c|}
\hline & Number of elements \\
\hline Triples & 335429988 \\
\hline Entities & 3226341 \\
\hline Classes & 10 \\
\hline Datasets & 53 \\
\hline
\end{tabular}

In the final Regulatory Circuits network, all the TFgene relations were qualified by a score (cf. Figure 2c). This score was the maximum of all the TF-gene relations scores obtained through either promoters (bottom part of Figure 2c) or enhancers (top part of Figure 2c). The intermediate score through a promoter was Confidence_Score $\times$ Rank_promoter. The intermediate score through an enhancer was Confidence_Score $\times$ Weight_Distance $\times$

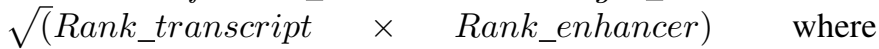
Rank_transcript is the max of the transcript promoters ranks.

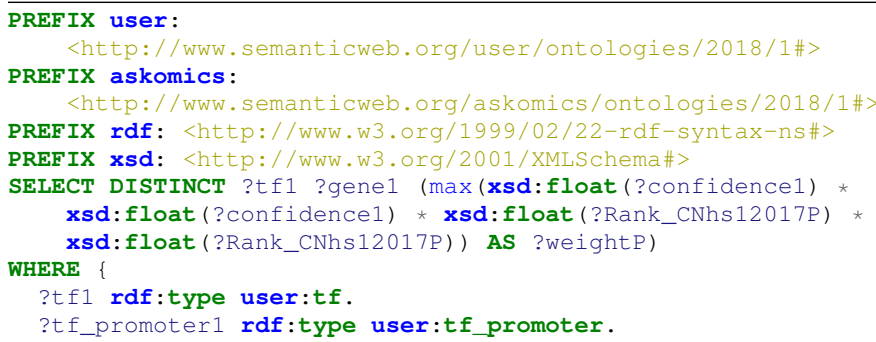

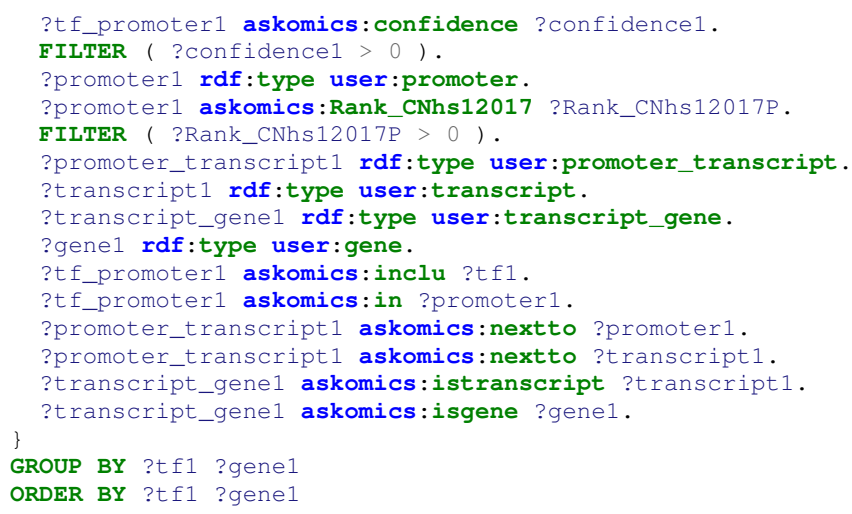

With our structured data we could extend our queries to compute the intermediate promoter and enhancer-related scores. SPARQL queries do not support square root, but could easily be devised to compute the square of the previously presented scores. For enhancers, although we could have written queries that compute Rank_transcript on the fly (and recompute it for each transcript every time a promoter is considered), we took advantage of the intermediary files where Rank_transcript values were already provided, and added these pre-computed Rank_transcript values to our RDF model. The resulting query for computing the score could then use directly the Confidence_Score $\times$ Weight_Distance $\times$

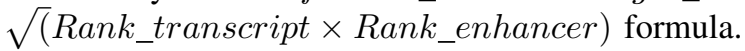

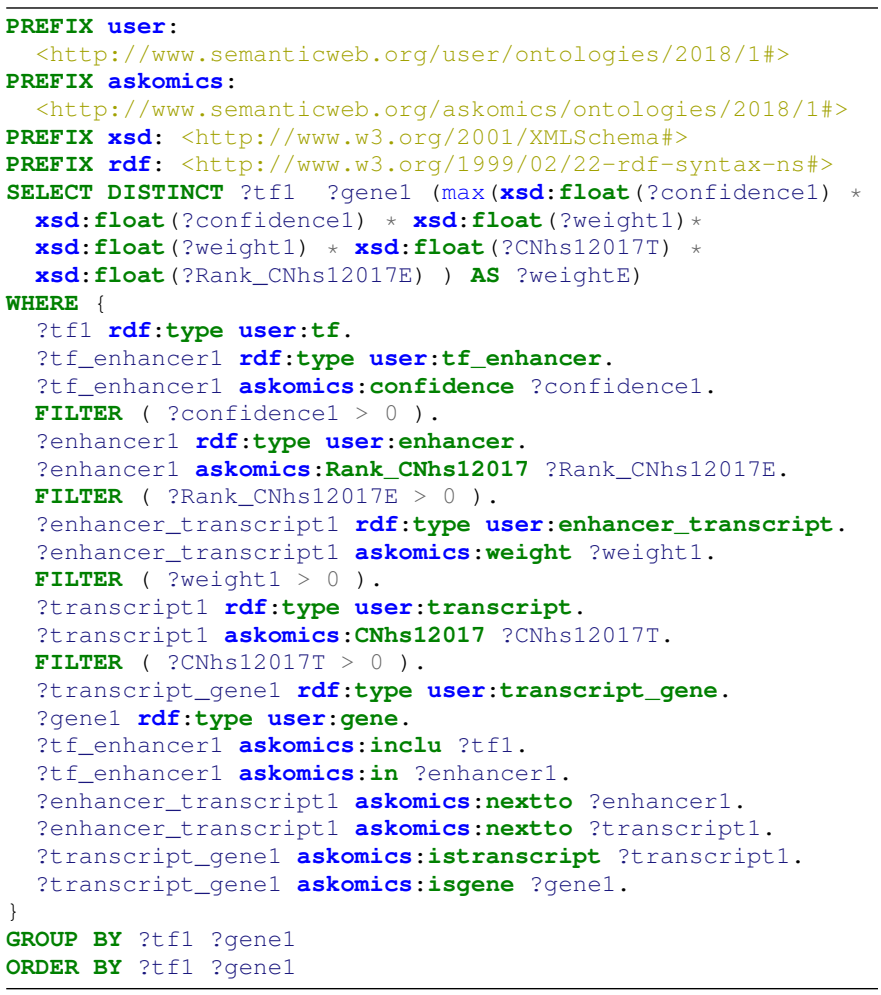

We then computed the score for TF-gene relations as the square root of the maximum of both the promoter and enhancer queries.

Overall, the complete Regulatory Circuits pipeline producing both TF-gene relations and their associated scores could be 
performed by 2 SPARQL queries. Theses queries were rather simple and involved 7 kinds of entities and 6 relations.

All queries in this section were based on the CNhs12017 sample of Regulatory Circuits and can be extended to other tissues by changing the sample name in the queries. The full list of tissue samples and their descriptions is given in the supplementary data file nmeth.3799-S2.xlsx from Regulatory Circuits $^{\dagger}$. A sub-list of samples names is given in Table IV in the following section.

\section{E. Performances}

Performance-wise, Table IV shows that all queries times ranged from 4.49 seconds for the fastest and 537.32 seconds ( 9 minutes) for the longest. On the 3.232 queries (4 queries for each 808 samples) only 124 had an execution time over 90 seconds. Each of the 4 queries have been performed on the 808 different samples of the dataset. This have been automated by using the python SPARQLwrapper library and feeding it the list of all different sample names.

TABLE IV: Queries' execution time (in seconds) for some of the 808 samples. They were run on the SPARQL end-point https://regulatorycircuits-rdf.genouest.org/sparql. The means are over the 808 samples.

\begin{tabular}{|c|c|c|c|c|}
\hline $\begin{array}{c}\text { Sample } \\
\text { name }\end{array}$ & $\begin{array}{c}\text { Queries for TF-relation based on: (in seconds) } \\
\text { all }>\mathbf{0}\end{array}$ & $\begin{array}{c}\text { Enhancers } \\
\text { all }>\mathbf{0}\end{array}$ & $\begin{array}{c}\text { Promoters } \\
\text { \& Score }\end{array}$ & $\begin{array}{c}\text { Enhancers } \\
\text { \& Score }\end{array}$ \\
\hline CNhs12017 & 19.310 & 16.359 & 31.319 & 20.515 \\
\hline CNhs13465 & 18.062 & 50.649 & 28.630 & 70.771 \\
\hline CNhs10629 & 23.631 & 20.755 & 37.505 & 27.434 \\
\hline CNhs11750 & 16.519 & 5.437 & 26.650 & 6.915 \\
\hline CNhs13195 & 16.138 & 26.339 & 28.377 & 38.867 \\
\hline CNhs13492 & 18.159 & 44.711 & 25.378 & 66.119 \\
\hline CNhs11771 & 22.666 & 13.026 & 30.768 & 16.451 \\
\hline CNhs12347 & 17.099 & 9.029 & 28.260 & 11.861 \\
\hline CNhs11047 & 21.361 & 35.926 & 35.727 & 49.122 \\
\hline CNhs12075 & 17.775 & 10.570 & 26.353 & 12.648 \\
\hline CNhs13099 & 16.105 & 9.275 & 24.457 & 12.225 \\
\hline CNhs12569 & 20.792 & 15.234 & 32.980 & 19.403 \\
\hline CNhs10636 & 28.768 & 51.718 & 42.956 & 75.017 \\
\hline CNhs11869 & 19.686 & 14.204 & 29.645 & 19.136 \\
\hline$\ldots$ & $\ldots$ & $\ldots$ & $\ldots$ & $\ldots$ \\
\hline Fastest & $\mathbf{1 2 . 0 6 4}$ & $\mathbf{4 . 4 8 7}$ & $\mathbf{1 8 . 0 5 4}$ & $\mathbf{4 . 7 3 4}$ \\
\hline Slowest & $\mathbf{1 4 8 . 2 3 2}$ & $\mathbf{3 2 9 . 6 5 5}$ & $\mathbf{2 1 7 . 8 0 6}$ & $\mathbf{5 3 7 . 3 1 9}$ \\
\hline Mean & $\mathbf{2 7 . 1 8 9}$ & $\mathbf{3 2 . 0 6 0}$ & $\mathbf{4 2 . 5 0 0}$ & $\mathbf{4 3 . 7 9 8}$ \\
\hline
\end{tabular}

We chose to have two distinct queries to retrieve TF-gene relations score, and to process their results to keep the maximal score instead of an unique query which would result in longer execution time.

\section{DISCUSSION}

Our approach consisted in structuring the data and results of a systems biology study as a RDF dataset. Our experience was that reusing the 21 raw and intermediary files from Regulatory Circuits required an in-depth analysis of their structure and of the documentation. We produced a RDF

$\dagger^{\dagger}$ https://media.nature.com/original/nature-assets/nmeth/journal/v13/n4/ extref/nmeth.3799-S2.xlsx model (Figure 3) of Regulatory Circuits that provides a unified access to their networks which are currently spread in 394 cell types and tissue-specific files, statically grouped into 32 highlevel regulatory networks. This RDF model saves future users from having to manually reproduce the integration effort. Our results showed that once the relations and ranks had been precomputed, the Regulatory Circuits analysis pipeline could be formalized as two SPARQL queries. We argue that this unified RDF dataset makes querying and reuse in other studies easier.

Even if the structure of our RDF model (Figure 3) is fairly simple, the Regulatory Circuits dataset is rather large (more than 300 millions triples, cf. Table III). Despite the size, SPARQL querying performances were of a few seconds (Table IV).

The Regulatory Circuits pipeline relies on raw data as well as external resources such as Ensembl that are regularly updated. To accomodate these updates, the original Regulatory Circuit data structure requires to update some raw files, regenerate the intermediary files that depend on them and run the pipeline. With our approach, these third-party updates can easily be propagated to our RDF model by running the SPARQL queries.

The RDF version of Regulatory Circuits allows a finegrained exploration of the relations between entities (transcription factors, enhancers, promoters, transcripts and genes) involved in regulation mechanisms. For example, it allows to differentiate the relations involving enhancers from the ones involving promoters (e.g. for taking into account that promoters relations are more reliable). Similarly, it allows to differentiate between the binding motifs of a single transcription factor or to consider transcription factors from a specific family that usually share similar binding site motifs.

The RDF version of Regulatory Circuits can also be extended with user-specific data, which increases flexibility. For example, if users have expression data of additional tissues, a new set of regulatory regions or binding data for an undescribed transcription factor, they can update the current model to add their new data. Depending on the type of data it may require pre-processing, to fit with Regulatory Circuits current dataset. Users can also import new data not present in the current data structure by following the rules described in Section III. This will require to extend the RDF graph (Figure 3), which is straightforward in RDF.

Following the Linked Data approach, we used the Ensembl identifiers for genes and transcripts. Federated SPARQL queries can then be used to combine information for Regulatory Circuits with information from Ensembl (e.g. variants, associations with diseases, or annotations).

Our approach is rather generic and should be easily transposed to other studies than Regulatory Circuits for which the analysis pipeline follows relations and performs simple arithmetic functions, such as parts of the ENCODE or Roadmap Epigenomics databases. More in-depth analyses (e.g. statistical) are beyond SPARQL expressivity and should be addressed by pre-processing. 


\section{CONClusion}

Life Science current standardization and integration efforts increasingly rely on Semantic Web technologies. They are currently directed towards reference data and knowledge bases. We hypothesized that applying the same approach to original studies would improve the results reproducibility, their maintenance and their reuse for advancing other studies. We considered the Regulatory Circuits case-study. We surveyed the 394 original data files and proposed an unified RDF data model. We showed that the Regulatory Circuits analysis pipeline can be formalized as two SPARQL queries and that the performances were acceptable. Overall, this unified RDF dataset makes querying and reuse in other studies easier.

\section{ACKNOWLEDGMENT}

Marine Louarn is supported by a joint INSERM-INRIA "Digital Health" PhD grant.

We acknowledge the GenOuest bioinformatics core facility (https://www.genouest.org) for providing the computing infrastructure.

\section{REFERENCES}

[1] M. Y. Galperin, D. J. Rigden, and X. M. Fernández-Suárez, "The 2015 nucleic acids research database issue and molecular biology database collection," Nucleic acids research, vol. 43, no. D1, pp. D1-D5, 2015.

[2] L. D. Stein, "Integrating biological databases," Nature Reviews Genetics, vol. 4, no. 5, p. 337, 2003.

[3] A. Kalderimis, R. Lyne, D. Butano, S. Contrino, M. Lyne, J. Heimbach et al., "InterMine: extensive web services for modern biology," Nucleic Acids Res., vol. 42, no. Web Server issue, pp. W468-472, Jul 2014.

[4] D. Smedley, S. Haider, S. Durinck, L. Pandini, P. Provero, J. Allen et al., "The BioMart community portal: an innovative alternative to large, centralized data repositories," Nucleic Acids Res., vol. 43, no. W1, pp. W589-598, Jul 2015.

[5] T. Berners-Lee and J. Hendler, "Publishing on the semantic web," Nature, vol. 410, no. 6832, pp. 1023-1024, Apr 2001.

[6] P. L. Whetzel, N. F. Noy, N. H. Shah, P. R. Alexander, C. Nyulas, T. Tudorache, and M. A. Musen, "BioPortal: enhanced functionality via new web services from the national center for biomedical ontology to access and use ontologies in software applications," Nucleic acids research, vol. 39, no. Web Server issue, pp. W541-W545, 2011.

[7] S. Jupp, J. Malone, J. Bolleman, M. Brandizi, M. Davies, L. Garcia et al., "The EBI RDF platform: linked open data for the life sciences," Bioinformatics, vol. 30, no. 9, pp. 1338-1339, May 2014.

[8] C. Bizer, T. Heath, and T. Berners Lee, "Linked data-the story so far," International Journal on Semantic Web and Information Systems, vol. 5 , no. 3, pp. 1-22, 2009.

[9] K. M. Livingston, M. Bada, L. E. Hunter, and K. Verspoor, "Representing annotation compositionality and provenance for the semantic web," Journal of biomedical semantics, vol. 4, p. 38, 2013.

[10] M. D. Wilkinson, M. Dumontier, I. J. J. Aalbersberg, G. Appleton, M. Axton, A. Baak et al., "The fair guiding principles for scientific data management and stewardship," Scientific data, vol. 3, p. 160018, 2016.

[11] A. Rodríguez-Iglesias, A. Rodríguez-González, A. G. Irvine, A. Sesma, M. Urban, K. E. Hammond-Kosack, and M. D. Wilkinson, "Publishing fair data: An exemplar methodology utilizing phi-base," Frontiers in plant science, vol. 7, p. 641, 2016.

[12] M. Brandizi, A. Singh, C. Rawlings, and K. Hassani-Pak, "Towards FAIRer biological knowledge networks using a hybrid linked data and graph database approach," Journal of integrative bioinformatics, vol. 15, no. 3,2018 , in press

[13] D. Marbach, D. Lamparter, G. Quon, M. Kellis, Z. Kutalik, and S. Bergmann, "Tissue-specific regulatory circuits reveal variable modular perturbations across complex diseases," Nature methods, vol. 13, no. 4, p. $366,2016$.
[14] E. P. Consortium et al., "An integrated encyclopedia of dna elements in the human genome," Nature, vol. 489, no. 7414, p. 57, 2012.

[15] M. B. Gerstein, A. Kundaje, M. Hariharan, S. G. Landt, K.-K. Yan, C. Cheng et al., "Architecture of the human regulatory network derived from encode data," Nature, vol. 489, no. 7414, p. 91, 2012.

[16] M. Lizio, J. Harshbarger, H. Shimoji, J. Severin, T. Kasukawa, S. Sahin et al., "Gateways to the fantom5 promoter level mammalian expression atlas," Genome biology, vol. 16, no. 1, p. 22, 2015.

[17] R. Andersson, C. Gebhard, I. Miguel-Escalada, I. Hoof, J. Bornholdt, M. Boyd et al., "An atlas of active enhancers across human cell types and tissues," Nature, vol. 507, no. 7493, p. 455, 2014.

[18] M. Skipper, A. Eccleston, N. Gray, T. Heemels, N. Le Bot, B. Marte, and U. Weiss, "Presenting the epigenome roadmap," Nature, vol. 518 , p. 313, 2015.

[19] A. Kundaje, W. Meuleman, J. Ernst, M. Bilenky, A. Yen, A. HeraviMoussavi et al., "Integrative analysis of 111 reference human epigenomes," Nature, vol. 518, no. 7539, p. 317, 2015.

[20] V. S. Malladi, D. T. Erickson, N. R. Podduturi, L. D. Rowe, E. T. Chan, J. M. Davidson et al., "Ontology application and use at the encode dcc," Database, vol. 2015, 2015.

[21] I. Abugessaisa, H. Shimoji, S. Sahin, A. Kondo, J. Harshbarger, M. Lizio et al., "Fantom5 transcriptome catalog of cellular states based on semantic mediawiki," Database, vol. 2016, 2016.

[22] C. Maumet, T. Auer, A. Bowring, G. Chen, S. Das, G. Flandin et al., "Sharing brain mapping statistical results with the neuroimaging data model," Scientific data, vol. 3, p. 160102, 2016. 